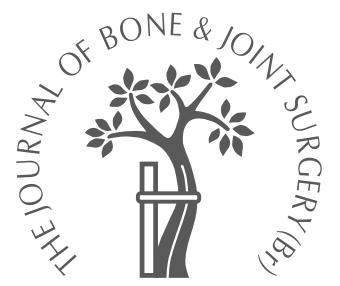

D. P. Gwynne Jones,

B. F. Hodgson,

N. A. Hung

From the Dunedin Hospital, Dunedin, New Zealand

\title{
Bilateral, uncemented total hip arthroplasty in osteopetrosis
}

\author{
Bilateral, uncemented hip replacements were performed on a 45-year-old woman with \\ autosomal dominant osteopetrosis. The hips showed degenerative changes and protrusio \\ acetabuli. Difficulties were encountered especially during preparation of the femoral canal. At \\ ten-year follow-up she has an excellent clinical and radiological result with no sign of osteolysis. \\ Uncemented hip replacement, while technically demanding, can be successful in the \\ intermediate term for patients with this condition.
}

Osteopetrosis or Marble Bone disease is an uncommon condition. The sex-linked recessive form is associated with childhood problems and early death. However, the autosomal dominant form is compatible with a normal life span, and as many as $40 \%$ of patients may remain asymptomatic. ${ }^{1,2}$ Bollerslev and Mosekilde ${ }^{1}$ described two forms of autosomal dominant osteopetrosis (ADO). In Type 1 , there is increased thickness of the cranial vault, diffuse osteosclerosis of the lumbar spine and pelvis and symmetrical, long-bone involvement. Type 2 shows more basal skull involvement, a 'rugger jersey' spine and 'endobones' within the pelvis. Type 2 patients are more prone to fractures which are difficult to treat.

Degenerative changes may occur in the hip and knee after the fifth decade, sometimes in the presence of deformity, especially coxa vara, ${ }^{3,4}$ and sometimes without..$^{5-10}$ There have been several early reports of hip arthroplasty for this condition, but we believe that this is the first report of an uncemented total hip arthroplasty (THA) in osteopetrosis.

FRACS, Senior Lecturer and Consultant Orthopaedic Surgeon

B. F. Hodgson, FRACS, Consultant Orthopaedic

Surgeon

N. A. Hung, FRCPA, Consultant Pathologist Department of Orthopaedic Surgery, Dunedin Hospital, Great King Street, Dunedin, New Zealand.

Correspondence should be sent to Mr D. P. Gwynne Jones.

(C)2004 British Editorial Society of Bone and Joint Surgery doi:10.1302/0301-620X.86B2. $14354 \$ 2.00$

$\mathrm{J}$ Bone Joint Surg $[\mathrm{Br}]$ 2004;86-B:276-8. Received 26 March 2003;

Accepted after revision

26 June 2003 cut and the cut surface showed no obvious medullary canal. A $52 \mathrm{~mm}$ CLS Expansion cup (Centerpulse Orthopedics, Munsinger, Switzerland) was inserted into the acetabulum after routine power reaming. Drills were used to create a medullary canal breaking two drill bits in the process. The femur was then sequentially reamed with power reamers and hand-held conical reamers to allow the insertion of a $14 \mathrm{~mm}$ Wagner cone prosthesis (Centerpulse Orthopedics) which has a roughblasted, titanium, conical stem with fins. There were no post-operative complications.

Histopathological examination of the femoral head revealed a thickened cortex and variably thickened bony trabeculae. In some areas, the trabeculae were markedly widened with both a woven and lamellar pattern, and islands of pale staining, alcianblue positive, myxoid material (Fig. 2) which may represent the remnant cartilaginous bridges which are typical of osteopetrosis. Delicately vascularised adipose tissue replaced haemopoietic marrow. Osteoclasts were seen in neither histological nor electronmicrographic sections. These histological features indicate abnormal bone remodelling and, when taken in conjunction with the clinical and radiological findings, are consistent with ADO Type 1 as described by Bollerslev and Mosekilde. ${ }^{1}$ The articular surface was eburnated and any remaining articular cartilage was fibrillated, as is seen in osteoarthritis.

At two-year follow-up, tomography (Fig. 3) suggested bone ongrowth with trabecular lines seen running from the threads of the cup. The HHS was 93 (pain 40, function 44). At the latest follow-up (ten years), she continues to have a good result with an HHS of 91 (pain 40, function 42), an Oxford-12 item hip score of 25, and a WOMAC score of 60 (pain 12, motion 5, function 43). Her short-form (SF)-12 physical score is 41.41 and her mental score is 42.20. She has residual discomfort from her lumbar spine but is satisfied with the results of her hip arthroplasties. The latest radiograph (Fig. 4) 


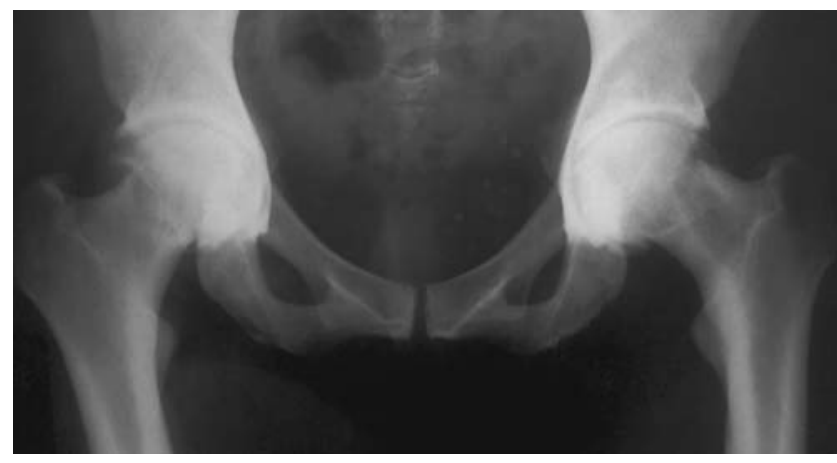

Fig. 1a

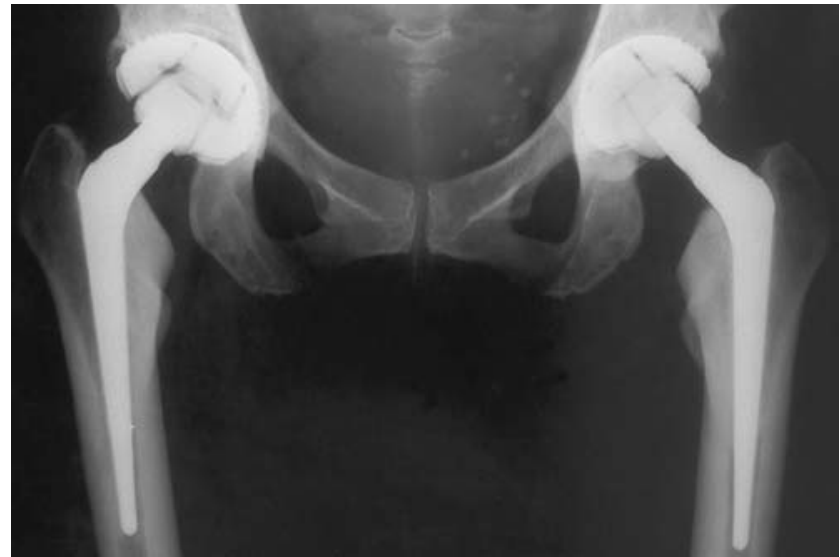

Fig. 1b

Fig. 1a - An anteroposterior (AP) radiograph of the pelvis showing dense sclerotic bone of osteopetrosis, degenerative changes in both hips and protrusio acetabuli. Fig. $1 \mathrm{~b}-\mathrm{An}$ AP radiograph of the pelvis, two years after surgery.

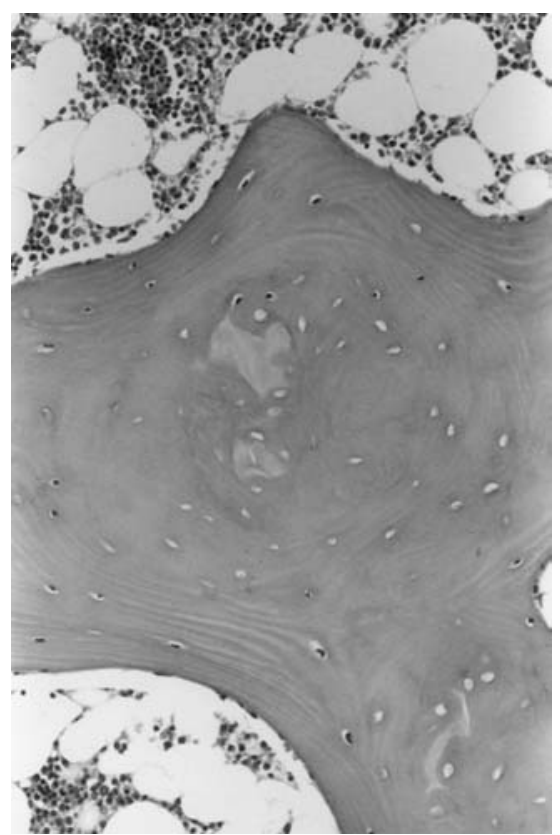

Fig. 2

Photomicrograph of the femoral head showing markedly widened bony trabeculae with central islands of pale staining myxoid material (haematoxylin and eosin $x$ 78)

shows apparently solid fixation of the components with no osteolysis. There has been minimal remodelling of the canal created distal to the femoral stem.

\section{Discussion}

The gene disorders in osteopetrosis result in decreased osteoclastic resorption of bone. Where present, osteoclasts lack the usual ruffled border that is important in bone resorption. The abnormal osteoclastic function results in very hard bone with thickened trabeculae. Coxa vara is a well-recognised complication of osteo-

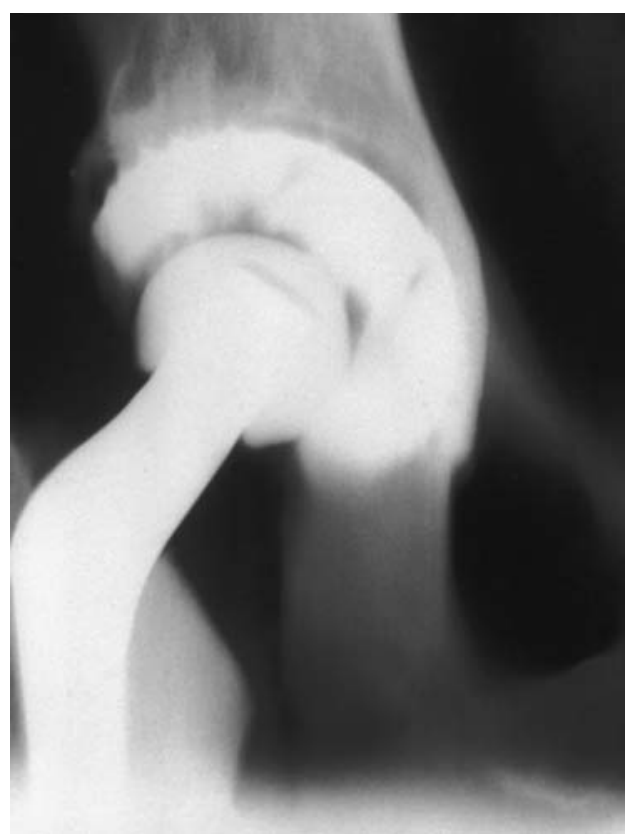

Fig. 3

A tomogram of the right hip at two years showing bone trabeculae running from the titanium threads of the Expansion cup. petrosis with most cases developing during childhood, ${ }^{2,10}$ sometimes after fracture of the femoral neck or subtrochanteric region. ${ }^{3}$

Degenerative changes often occur after the age of $40^{5-10}$ in the absence of deformity. Articular cartilage is not affected by the disorder, and it has therefore been suggested that the degeneration occurs because of the hard unyielding subchondral bone. ${ }^{6,8}$ Protrusio has not been reported previously. We speculate that it may have occurred because the acetabular bone was relatively less hard than the femoral head. 


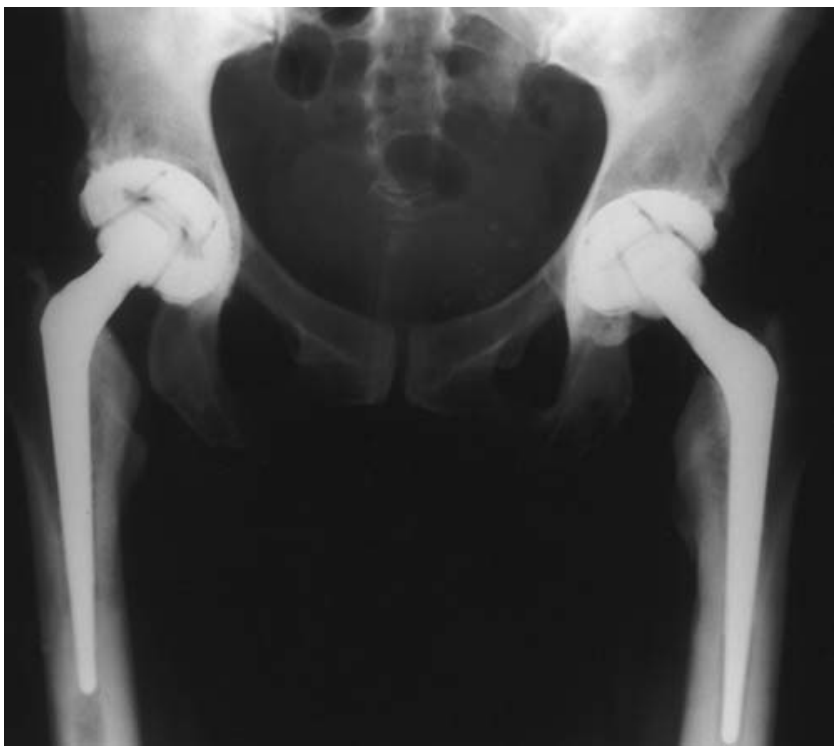

Fig. 4

A radiograph 10 years after surgery showing no osteolytes and minimal remodelling of the medullary canal distal to the femoral stem.

There are several case reports of hip and knee arthroplasty with follow-up ranging from six months to six years. ${ }^{3-10}$ Technical difficulty has been experienced especially on the femoral side. Drills and high-speed burrs, guided by fluoroscopy, have been used to help create a medullary canal. Previously, cemented components have been used. Matsuno and Katayama ${ }^{4}$ used a press-fit acetabular component with screw fixation, since it would be difficult to obtain good quality bone-cement interface, but considered that to use a cementless femoral component risked fracturing the shaft. We found that the use of the Expansion cup allowed more controlled insertion and may reduce the risk of acetabular fracture. The shape and small size of the Wagner cone prosthesis allowed power reaming of the femur and avoided downsizing the component to allow a cement mantle. Poor cement-bone penetration would be expected to occur in the dense sclerotic bone.

Fracture healing occurs slowly in osteopetrosis and slow bony ongrowth to the titanium prosthesis would be expected, which appears to have occurred in our case. Serial radiographs over a period of 10 years show no progressive osteolysis despite a high level of activity. There has been minimal remodelling of the femur where the medullary canal was reamed out. The osteoclast has a central role in osteolysis due to particulate debris and patients with osteopetrosis may have an inhibited process of osteolysis.

In summary, we believe that this is the first reported case of fully cementless THA in osteopetrosis. At intermediate follow-up of ten years, the clinical and radiological results are good. Protrusio acetabuli has not previously been described in this condition.

No benefits in any form have been received or will be received from a commercial party related directly or indirectly to the subject of this article.

\section{References}

1. Bollerslev J, Mosekilde L. Autosomal dominant osteopetrosis. Clin Orthop 1993; 294:45-51.

2. Armstrong DG, Newfield HJT, Gillespie R. Orthopaedic management of osteopetrosis: results of a survey and review of the literature. J Pediatr Orthop 1999;19: 122-32.

3. Ashby ME. Total hip arthroplasty in osteopetrosis: a report of two cases. Clin Orthop 1992;276:214-21

4. Matsuno T, Katayama N. Osteopetrosis and total hip arthroplasty: report of two cases. Int Orthopaedics (SICOT) 1997;21:409-11.

5. Janecki CJ, Nelson CL. Osteoarthritis associated with osteopetrosis treated by total hip replacement arthroplasty: report of a case. Cleve Clinic 0 1971;38:169-77.

6. Cameron HU, Dewar FP. Degenerative osteoarthritis associated with osteopetrosis. Clin Orthop 1977;127:148-9.

7. Milgram JW, Jasty M. Osteopetrosis: a morphological study of twenty-one cases. J Bone Joint Surg [Am] 1982;64-A:912-29.

8. Casden AM, Jaffe FF, Kastenbaum DM, Bonar SF. Osteoarthritis associated with osteopetrosis treated by total knee arthroplasty: report of a case. Clin Orthop 1989; 247:202-7.

9. Siegal A, Delling G. Total hip joint endoprosthesis in osteoporosis. Chirug (1992); 63:984-7.

10. Shapiro F. Osteopetrosis: current clinical considerations. Clin Orthop 1993:294: 34-44. 